\title{
Perinatal outcome in terminal oligohydramnios
}

\author{
Rajeshree Tukaram Patil', Renuka Vivekanand Patil' \\ ${ }^{1}$ Associate Professor, Department of Obstetrics and Gynecology, Government Medical College, Gondia, \\ ${ }^{2}$ Research Assistant, Indira Gandhi Government Medical College, Nagpur
}

Background of the Study: Amniotic fluid or liquor, the fluid around the developing fetus serves various functions. A balance of formation and reabsorption of this fluid has to be maintained. The amniotic fluid volume (AFV) undergoes changes during pregnancy. Oligohydramnios is a condition in which the AFV decreases and is associated with conditions like renal agenesis, placental dysfunction, congenital anomalies, anemia, PIH, hypoxia. Its effects depend on the duration of pregnancy. But when AFV decreases terminally, the chances of having a normal baby are more. Aims and Objective: This study was aimed to find the perinatal outcome in terms of LSCS and instrumental deliveries, needs for induction, neonatal mortality, low apgars, meconium staining, NICU admissions in women with oligohydramnios occurring after 34 weeks of pregnancy. Materials and Methods: A prospective observational study included women with singleton pregnancy admitted with intact membranes after 34 weeks. AFV was calculated by USG. Results: Less number of women had AF Index less than $5 \mathrm{~cm}$ but as AFV decreases risk of LSCS increases and spontaneous onset of labor ends mostly with vaginal deliveries. Amniotic fluid Index less than $5 \mathrm{~cm}$ increases the risk of low Apgar, septicaemia and neonatal mortality. Although Apgar was low at $1 \mathrm{~min}$, it improved at 5 min suggesting a good adaption outside utero. Conclusion: Low AF Index (as determined by USG)in last trimester was associated with increased risk of adverse perinatal outcome like operative delivery, fetal distress, NICU, low apgar, septicemia, neonatal mortality. Here, Doppler can relieve obstetrician stress in decision making and amnioinfusion in selected cases can reduce operative morbidity. Parameters like risk of septicemia and effects in extremes of maternal age need to be evaluated further in a larger number of cases.

Access this article online

Website:

http://nepjol.info/index.php/AJMS

DOI: 10.3126/ajms.v8i6.18333

E-ISSN: 2091-0576

P-ISSN: 2467-9100

Key words: terminal oligohydramnios, perinatal, apgars, amniotic fluid index, LSCS

\section{INTRODUCTION}

Amniotic fluid or liquor is the fluid around the developing fetus. When it becomes $<5^{\text {th }}$ percentile or less than $500 \mathrm{ml}$ at more than 34 weeks, is termed as oligohydramnios. The amniotic fluid serves various important functions including cushioning the fetus, maintaining temperature within the uterus, prevention of cord compression, allowing fetal movements and also aids the digestive and respiratory systems of the fetus to develop because the fetus "swallows and excretes" and "inhales and exhales" this fluid.
Circulation is initially as a transudate from maternal blood. There are osmotic and hydrostatic forces across the vessel walls till 14 wks. At about 20 weeks fetal swallowing and urination starts.

A balance between input and output or formation and reabsorption of fluid is maintained. ${ }^{1}$

The AFV (Amniotic Fluid Volume) undergoes characteristic changes with gestation. It progressively rises from $10-20 \mathrm{ml}$ at 10 weeks gestation to $\sim 250 \mathrm{ml}$ at 16 weeks reaching $\sim 800 \mathrm{ml}$ at 33 weeks and reaching a plateau $\sim 1000 \mathrm{ml}$ 
at 38 - 39 weeks before finally decreasing to $\sim 800 \mathrm{ml}$ at 40 weeks. ${ }^{2-5}$ Although the etiology is complex, it is mostly associated with conditions like renal agenesis, placental dysfunction, congenital anomalies, under nutrition, PIH, hydration anemia and hypoxia.

The effects of oligohydramnios depend largely on the stage of pregnancy at which it occurs. ${ }^{6-9}$ For example, in $1^{\text {st }}$ trimester there can be serious consequences like birth defects. In $2^{\text {nd }}$ trimester problems like miscarriage, premature births, IUD, muscle and limb defects can occur. In $3^{\text {rd }}$ trimester there can be fetal distress, RDS, IUD, fetal compression syndrome/ pulmohypoplasia / amniotic band syndrome. But, when oligohydramnios appears terminally in pregnancy, the chances of having a normal baby are increased. ${ }^{10-12}$

With this background, the present study was taken up with the aimto find out the perinatal outcome in mothers showing oligohydramnios at $>34$ weeks gestation (terminal) in terms of: LSCS and instrumental deliveries, need for induction, neonatal mortality, low apgars, meconium staining, NICU admissions, septicemia, IUD, modifications in management.

\section{Ethical issues}

The participants were informed about the nature of study, the importance of doing USG, notification of the birth outcome and the ability to withdraw from the study at any point of time without any hindrance in management. Their participation was voluntary and confidentiality was assured.

\section{MATERIALS AND METHODS}

A prospective observational study was carried out for 3 months that included women having singleton pregnancy and admitted with oligohydramnios detected on USG at $>34$ weeks with intact membranes. These women in whom clinically oligohydramnios was suspected were subjected to USG for confirmation. The gestational age of women was confirmed from earlier ANC follow-ups and early USG (this also ensured that they did not have oligohydramnios earlier) -thus only low risk terminal oligohydramnios cases were included. Women with PROM, APH, uterine or fetal anomalies, maternal diseases like anemia, diabetes mellitus, heart disease, pregnancy induced hypertension, HD, PIH were excluded from the study. ${ }^{10}$

USG performed using Siemens sector probe $3.5 \mathrm{MHz}$ used to measure vertical depth in 4 quadrants. Precautions were taken to keep the probe perpendicular, no pressure was applied, no limbs or no cord were observed in the measured pockets. Qualitative estimation included interobserver/intra-observer variability which was avoided by using only one sonologist for the study. A four quadrant assessment done for the quantitative estimation. The AFI (Amniotic Fluid Index) which is the sum of four quadrants, was calculated as per Phelan as very low $/$ low/ normal/polywhich coincides with $<5 / 5-8 />8 / 18 \mathrm{~cm}^{13}$ Once oligohydramnios was detected the women were subjected to Doppler for determination of fetal well being. Accordingly, management (LSCS/Vaginal) was decided after doing $\mathrm{P} / \mathrm{V}$ examination. After birth, APGAR was noted at 1 and $5 \mathrm{~min}$, and follow-up was till day 15 of birth. Number of babies getting admitted in NICU and appearance of septicemia were recorded separately.

\section{RESULTS}

Incidence of oligohydramnios as calculated from the hospital admission register was 12.5/100 women getting admitted at 34 weeks and above. The total number of cases studied were 51, which included (1)33 women in 22-32 yrs age group.(2) 9 women in 18-21 yrs age group.

\section{Table 1: Comparison of vaginal vs LSCS in AFI $\leq \mathbf{5}$ (Group A)}

\begin{tabular}{llcccc}
\hline S. No. & Route of delivery & Mean \pm SD & T calculated & T tabulated & Level of significance $\mathbf{d}, \mathbf{f}$ \\
\hline 1 & 8 (vaginal) & $3.3125 \pm 3.0919$ & 0.0039 & 1.833 & $0.05,9$ \\
2 & 10 (LSCS) & $3.31 \pm 0.3324$ & & & \\
\hline
\end{tabular}

\begin{tabular}{|c|c|c|c|c|c|}
\hline S. No. & Number of patients & Mean $\pm S D$ & T calculated & $T$ tabulated & Level of significance $d, f$ \\
\hline 1 & 21 (vag) & $7.2905 \pm 0.7004$ & 1.4839 & 1.796 & $0.5,11$ \\
\hline 2 & 12 (LSCS) & $6.9 \pm 0.4308$ & & & \\
\hline
\end{tabular}

\begin{tabular}{|c|c|c|c|c|}
\hline AFI & Number of patients & Mean $\pm S D$ & T 0.5494 (calculated) & $\mathrm{T}$ (tabulated) \\
\hline$\leq 5 \mathrm{~cm} \mathrm{(A)}$ & 9 & $3.3111 \pm 1.0787$ & & \\
\hline$\geq 5 \mathrm{~cm}(\mathrm{~B})$ & 23 & $6.9870 \pm 0.5494$ & _9.6955 & 1.833 \\
\hline
\end{tabular}


(3) 5 women in 33 -38yrs age group. The total participating women were 51 .

Patient distribution was done as per Phelan ${ }^{2,13}$ after calculating the AFI. Women with AFI $\leq 5 \mathrm{~cm}$ were belonged Group A comprising of 18 women. Women with AFI $>5 \mathrm{~cm}$ (i.e., $5-8 \mathrm{~cm}$ ) were added to Group B, comprising of 33 women. Thus, being only terminal oligohydramnios, a lesser number of women had AFI < $5 \mathrm{~cm}$. The comparison of vaginal with LSCS in AFI $\leq 5$ (A) is shown in Table 1 and comparison between vaginal vs LSCS if AFI >5 (Group B) is shown in Table 2. More women had cesarean when AFI $<5 \mathrm{~cm}$ whereas number of women delivering vaginally was more when AFI $>5 \mathrm{~cm}$. Thus, increasing AFI is safer for vaginal delivery.

Table 3 presents the comparison between AFI $\leq 5$ and $>5$ for LBW i.e. baby weightless than or equal to $2.5 \mathrm{~kg}$. A total 32/51 i.e., $62.74 \%$ babies were LBW. Thus, low AFI is likely to be associated with LBW babies.

\section{Outcome with spontaneous labor}

A total of 9 women went into spontaneous labor. Of these 9 women, 4 women had AFI $\leq 5 \mathrm{~cm}$ and 5 women had AFI $>5 \mathrm{~cm}$. Eight of the total 9 women delivered vaginally while one of the 9 underwent LSCS. The effect of induction (baby weight $<2.5 \mathrm{~kg}$ ) is illustrated in Table 4. A total of 24 inductions were required for baby weight $<2.5 \mathrm{~kg}$, of which $75 \%$ delivered vaginally. Table 5 presents the indications of LSCS with AFI $<5 \mathrm{~cm}$, severe oligohydramnios was the main cause of LSCS.

Table 6 presents the relationship between maternal age and neonatal mortality. Total neonatal mortality observed in the study was $7(13.72 \%)$. As the maternal age moved to extremes, the neonatal mortality persisted. In group A (AFI $\leq 5 \mathrm{~cm}), 5$ neonatal deaths occurred. These included 3 babies of LSCS. One of these babies died of septicemia while 2 were neonatal deaths. Two babies delivered vaginally were IUD (Intra Uterine Death). In group B (AFI $>5 \mathrm{~cm}$ ), there were 2 deaths, 1 LSCS delivery which was a NICU admission had septicemia and died

\begin{tabular}{|c|c|c|c|}
\hline AFI index & $n=24$ & $\begin{array}{l}\text { Induced and had } \\
\text { vaginal delivery }\end{array}$ & $\begin{array}{l}\text { Induced and } \\
\text { had LSCS }\end{array}$ \\
\hline$A F I \leq 5 \mathrm{~cm}(\mathrm{~A})$ & 4 & 4 & 0 \\
\hline$A F I>5 \mathrm{~cm}(\mathrm{~B})$ & 20 & $14(63.6 \%)$ & $6(27.27)$ \\
\hline
\end{tabular}

on day10; 1 vaginal delivery was an IUD. Thus, as AFI decreases neonatal morbidity and mortality increases.

\section{APGAR}

A Total of 19/51(37.25\%) had low apgar $\leq 6$ at $1 \mathrm{~min}$. Sixtytwo pint seventy five percentage had apgar $>7$ at $5 \mathrm{~min}$.

Apgar at 1 min: When AFI was less than $5 \mathrm{~cm}, 9 / 18$ babies had a low Apgar of which 2 babies could not recover (neonatal mortality).

The number of NICU admissions is presented in Figure 1 and the number of septicaemia cases is presented in Figure 2. There were $4(7.8 \%)$ NICU admissions, of which 3 had AFI $<5 \mathrm{~cm}$. There were 3 cases of septicemia (5.88\%), of which 2 had AFI $<5 \mathrm{~cm}$. Conclusively, septicaemia and NICU admissions increase with decreasing AFI.

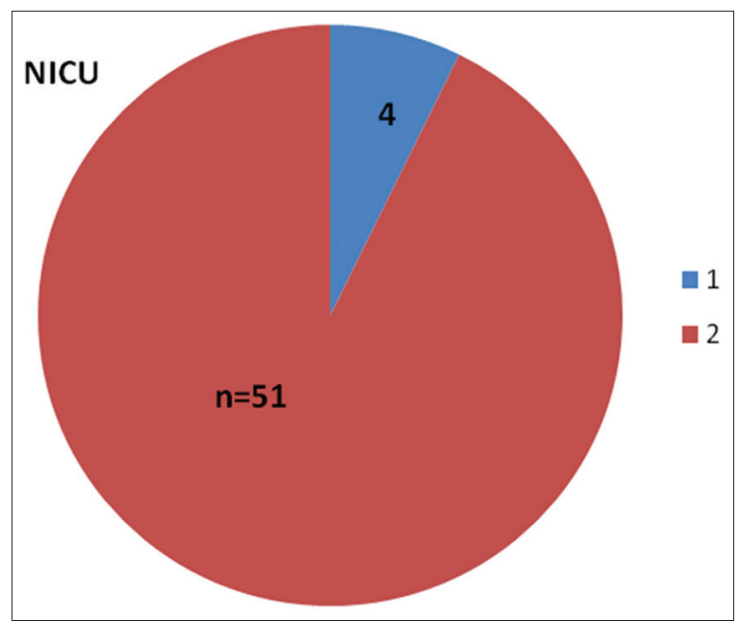

Figure 1: NICU Admissions

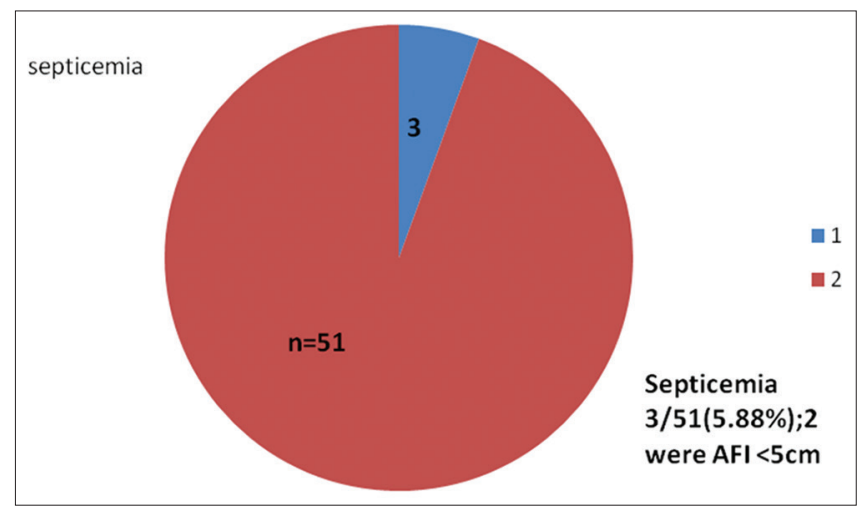

Figure 2: Percentage of Septicaemia cases

Table 5: Indications LSCS when AFI $\leq 5$ and AFI $>5$

\begin{tabular}{|c|c|c|c|c|c|}
\hline AFI & LSCS & Indications & & & $\%$ \\
\hline$\leq 5(A) n=18$ & 10 & 10 (severe oligo) & & & 55.55 \\
\hline$>5$ (B) $n=33$ & 12 & 6 (FD) & $3(\mathrm{FP})$ & 3 (Derranged doppler) & 36.36 \\
\hline
\end{tabular}




\begin{tabular}{lccc}
\multicolumn{4}{l}{ Table 6: Maternal age and neonatal mortality } \\
\hline Age & $\mathbf{1 8 - 2 1}$ & $\mathbf{2 2 - 3 2}$ & $\mathbf{3 3 - 3 8}$ \\
\hline Mortality & 1 & 5 & 1 \\
$\%$ & 14.2 & 71.42 & 14.2 \\
\hline
\end{tabular}

\section{DISCUSSION}

Although the incidence of oligohydramnios is $4.5 \%$ at term, the incidence in a tertiary referral hospital is higher. The effects ofoligohydramnios in extremes of age has not been studied extensively in the referred literature. ${ }^{14,15}$ In present study, the age range is wider but more patients are required to come to a conclusion for effects of oligohydramnios in extremes of age. According to Gumus, Koktener and Turhanthe rate of induction is high (73\%) in oligohydramnios. ${ }^{16}$ Manzanares et al found high operative delivery rate than spontaneous, with no difference in perinatal outcome. ${ }^{17}$ Leeman and Almond recommended the management be individualized based on parity / cx ripening. ${ }^{18}$ Crowley et al concluded that inductions are associated with an increased rate of LSCS along with statistically significant increase in meconium staining. ${ }^{19}$ Sarno et al reported an increased rate of LSCS for FD and one minute Apgar was $<7 .{ }^{20}$ In the present study, total inductions were $47 \%$ while $17.6 \%$ of subjects had spontaneous labor. Of the total inductions, 6 inductions ended up in LSCS for FD with meconium for 3 of them and baby weight less than $2.5 \mathrm{~kg}$. Hoddick et al reported that oligohydramnios was a poor predictor of baby weight less than $2.5 \mathrm{~kg}^{21}$ Perni et al found no significant relation between AFI and birth weight less than $2.5 \mathrm{~kg} .{ }^{22}$ Chamberlain et $\mathrm{al}^{23}$, Gumus, Koktener and Turhan ${ }^{16}$ reported that even borderline low AFI has a high incidence of baby weight less than $2.5 \mathrm{~kg}$. Banks and Miller $^{24}$ found an increased incidence of LBW (low birth weight) babies and meconium in borderline AFI. In Present study, baby weightless than $2.5 \mathrm{~kg}$ in both groups of AFI (Total $=62.7 \%$ ) suggests that the process of decreased nutrition (vasc) could have started earlier thereby giving more babies with less than $2.5 \mathrm{~kg}$, although oligohydramnios was detected as a terminal event.

\section{Apgar}

A low apgar at $5 \mathrm{~min}$ for borderline AFI was reported by some authors, but the results were not found to be statistically significant. ${ }^{6,15,16,24}$ Most of the babies having low apgar at $1 \mathrm{~min}$ have good apgar at $5 \mathrm{~min} .{ }^{25} \mathrm{Ghosh}$, Marsal and Gudmundsson ${ }^{26}$ observed a high incidence of low apgar at 1 and 5 min even in borderline AFI. In present study, although Apgar was low at $1 \mathrm{~min}$ (which may mean that the birth process was not well tolerated or it created stress) but it improved to normal at 5 min suggesting that they were well adapting to the new environment outside utero.

\section{$\mathrm{NICU}$}

Ghosh, Marsal and Gudmundsson ${ }^{25}$ and Gumus, Koktener and Turhan ${ }^{16}$ reported higher rates of NICU admissions in low AFI. Verma ${ }^{27}$ reported oligohydramnios was associated with more number of preterm deliveries, non-reactive NST, fetal distress and more NICU admissions. In the present study only $7.8 \%$ subjects had NICU admissions. However, most admissions were for AFI less than $5 \mathrm{~cm}$. Conclusively, a decrease in AFI is reflected by an increase in the rate of NICU admissions.

\section{Septicemia}

Septicemia in oligohydramnios has not been reported extensively in the referred literature. In the present study, septicemia was seen in total 3 cases (2 were in AFI less than $5 \mathrm{~cm}$ ) and all of the septicemia cases had a fatal outcome. However, more detailed studies are needed for any definitive conclusions.

\section{Limitations}

Effect of parity (previous vaginal deliveries) with respect to vaginal deliveries and inductions was not considered in this study. It might help the outcome by facilitating a fast labor.

DOPPLER was not done for most cases in the study, but a successful induction was seen with good Doppler indices in the present study.

\section{Plan of future research}

Effect of amnioinfusion in selected cases of oligohydramnios going in spontaneous labor can help to reduce LSCS morbidity.

\section{CONCLUSION}

- Low AFI in (terminal) last trimester is associated with increased risk of adverse perinatal outcome like increasing LSCS, FD, NICU admissions, low Apgar, deaths due to septicemia. AFI decreasing below $5 \mathrm{~cm}$ further increases the risk.

- Women with spontaneous onset of labor largely deliver vaginally (this aspect is not much reported in referred literature). This was seen even in mothers with severe oligohydramnios. Here the role of amnioinfusion for selected cases in competent hands can be helpful to reduce operative interferences.

- Doppler can relieve the obstetricians stress wherever vaginal delivery is anticipated or in women with spontaneous onset labor, even if AFI is less than $5 \mathrm{~cm}$.

- More studies are needed to evaluate the outcome on parameters like septicemia which can be a dangerous manifestation in LBW babies with sever oligohydramnios. 
- Outcome in extremes of maternal age also needs to be studied on a larger number of women.

\section{ACKNOWLEDGEMENTS}

Special Thanks for help in data collection to Dr Mohini Agrawal and Sheetal Paliwal for assisting in statistical analysis.

\section{REFERENCES}

1. Sherer, DM. A Review of Amniotic Fluid Dynamics and the Enigma of Isolated Oligohydramnios. American Journal of Perinatology 2002; 19(5), 253-266.

2. Phelan JP, Smith CV, Broussard P and Small M. Amniotic fluid volume assessment with the four-quadrant technique at 36-42 weeks' gestation. J Reprod Med 1987; 32 (7):540-542.

3. Khadilkar SS, Desai SS, Tayade SM and Purandare CN. Amniotic fluid index in normal pregnancy: an assessment of gestation specific reference values among Indian women. J Obstet Gynaecol Res 2003;29(3):136-141.

4. Magann EF, Nolan TE, Hess W, Martin RW, Whitworth NS and Morrison JC. Measurement of amniotic fluid volume: accuracy of ultrasonography techniques. Am J Obstet Gynecol1992;167 (6):1533-1537.

5. Boyd RL and Carter BS. Polyhydramnios and Oligohydramnios [online] 2002. eMedicine. [Cited 2003 Jun].Available from: URL:http://www.emedicine.com.

6. Baron C, Morgan MA and Garite TJ. The impact of amniotic fluid assessed intrapartum on perinatal outcome.Am J Obstet Gynecol 1994; 173 (1):167-174.

7. Elliott $\mathrm{PM}$ and Inman $\mathrm{WH}$. Volume of liquor amnio in normal and abnormal pregnancy. Lancet 1961 14;2(7207):835-840.

8. Sohaey R. Amniotic Fluid and the Umbilical Cord: The fetal Milieu and Lifeline. Semin Ultrasound CT MR 1998;19(4):355-369.

9. Halperin ME, Fong KW, Zalev $\mathrm{AH}$ and Goldsmith $\mathrm{CH}$. Reliability of amniotic fluid volume estimation from ultrasonograms: interobserver and intraobserver variation before and after the establishment of criteria. Am J Obstet Gynecol 1985;153:264-267.

10. Rutherford SE, Smith CV, Phelan JP, Kawakami K and Ahn MO. Four quadrant assessment of amniotic fluid volume, interobserver and intraobserver variation. J Reprod Med 1987;32: 587-589.

11. Sriya $R$ and Singhai $S$. Perinatal outcome in patients with amniotic fluid index less than $5 \mathrm{~cm}$. J Obstet and Gynecol of India 2001; 51(5):98-100.

12. Chaudhary R, Dhama V, Singh S and Singh M. Correlation of reduced amniotic fluid index with neonatal outcome. Int J Reprod Contracept Obstet Gynecol 2017;6(6):2401-2406.

13. Phelan JP, Ahn MO and Smith CV. Amniotic fluid measurement during pregnancy. J Reproductive medicine 1987;32:601-604.

14. Casey BM, McIntire DD, Bloom SL, Lucas MJ, Santos R, Twickler DM, et al. Pregnancy outcomes after antepartum diagnosis of oligohydramnios at or beyond 34 weeks' gestation. Am J Obstet Gynecol 2000; 182(4):909-912.

15. Phoolchandra, Kaur SP, Hans DK and Kapila AK. The impact of amniotic fluid volume assessment intrapartum on perinatal outcome. Obstet Gynecol Today 2000; 8:478-481.

16. Gumus II, Koktener A and Turhan NO. Perinatal outcomes of pregnancies with borderline amniotic fluid index. Arch Gynecol Obstet 2007; 276(1):17-19.

17. Manzanares S, Carrillo MP, González-Perán E, Puertas A and Montoya F. Isolated oligohydramnios in term pregnancy as an indication for induction of labor. J Matern Fetal Neonatal Med 2007;20(3):221-224.

18. Leeman $L$ and Almond $D$. Isolated oligohydramnios at term: is induction indicated? J Fam Pract 2005; 54(1):25-32.

19. Crowley P, O'Herlihy $C$ and Boylan P. The value of ultrasound measurement of amniotic fluid volume in the management of prolonged pregnancies. $\mathrm{Br} J$ Obstet Gynaecol 1984; 91(5):444-448.

20. Sarno AP, Ahn MO, Brar HS, Phelan JP and Platt LD. Intrapartum Doppler velocimetry, amniotic fluid volume, and fetal heart rate as predictors of subsequent fetal distress. I. An initial report. Am J Obstet Gynecol 1989;161(6 Pt 1):1508-1514.

21. Hoddick WK,Krishon B,Smith EO and Cotton DB. USG determination of qualitative amniotic fluid volume in IUGR. Reaaessment of 1 cm rule.Am J Obstet Gynecol 1984;149:758-762.

22. Perni SC, Predanic M, Cho JE, Kalish RB and Chasen ST. Association of amniotic fluid index with estimated fetal weight. $J$ Ultrasound Med 2004; 23(11):1449-52; quiz 1453.

23. Chamberlain PF, Manning FA, Morrison I, Harman CR and Lange IR. Ultrasound evaluation of amniotic fluid volume. I. The relationship of marginal and decreased amniotic fluid volumes to perinatal outcome. Am J Obstet Gynecol 1984;150(3):245-249.

24. Banks $\mathrm{EH}$ and Miller DA. Perinatal risks associated with borderline amniotic fluid index.Am J Obstet Gynecol1999; 180 (6):1461-1463.

25. Committee on obstetrical practices fetus and newborn. American Congress on Obstetrics and Gynecology, Number333, May2006.

26. Ghosh G, Marsal K and Gudmundsson S. Amniotic fluid index in low risk pregnancy as an admission test to the labor ward. Acta Obstetrica et Gynecologica Scandinavica 2002;81(9):852-855.

27. Verma M, Gupta S, Ahuja MV and Pratap C. Relationship of decreased amniotic fluid and perinatal outcome: A comparative study. Int J Res Med Sci 2016; 4(9):4073-4096.

\section{Authors Contribution:}

RTP- Concept of study, Study design, data collection, analysis and interpretation of data, Manuscript preparation; RVP- Data collection and Final Approval.

Orcid ID:

Dr. Rajeshree Tukaram Patil: (1) http://orcid.org/0000-0002-7619-242X

Dr. Renuka Vivekanand Patil: (1) http://orcid.org/0000-0002-2903-084X

Source of Support: Nil, Conflict of Interest: None declared. 